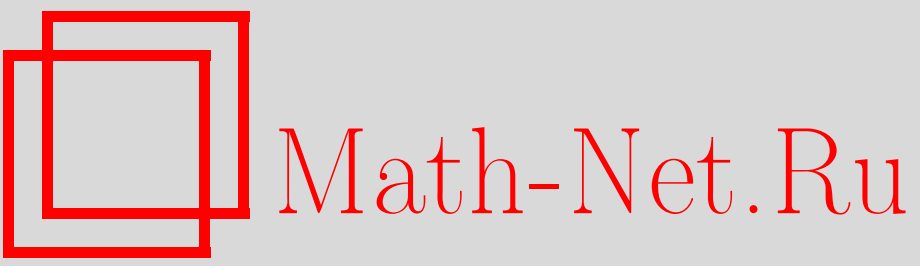

Е. В. Серегина, М. А. Степович, В. В. Калманович, О нахождении моментных функций стохастического процесса теплопроводности с использованием проекционного метода, Итоги науки и техн. Сер. Соврем. мат. и ее прил. Темат. обз., 2021, том 192, 102-110

DOI: https://doi.org/10.36535/0233-6723-2021-192-102-110

Использование Общероссийского математического портала Math-Net.Ru подразумевает, что вы прочитали и согласны с пользовательским соглашением

http://www.mathnet.ru/rus/agreement

Параметры загрузки:

IP : 54.210 .77 .194

26 апреля 2023 г., 16:48:15 


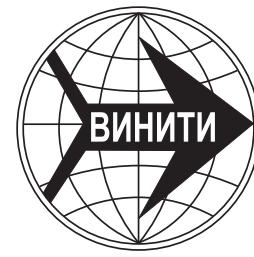

ИТОГИ НАУКИ И ТЕХНИКИ.

Современная математика и ее приложения.

Тематические обзоры.

Том 192 (2021). С. 102-110

DOI: $10.36535 / 0233-6723-2021-192-102-110$

УДК 517.584

\title{
О НАХОЖДЕНИИ МОМЕНТНЫХ ФУНКЦИЙ СТОХАСТИЧЕСКОГО ПРОЦЕССА ТЕПЛОПРОВОДНОСТИ С ИСПОЛЬЗОВАНИЕМ ПРОЕКЦИОННОГО МЕТОДА
}

\author{
(C) 2021 г. В. В. СЕРЕГИНА, М. А. СТЕПОВИЧ, В. В. КАЛМАНОВИЧ
}

\begin{abstract}
АннотАция. Изложены некоторые результаты использования проекционного метода наименьших квадратов для нахождения моментных функций первого и второго порядка решения уравнения теплопроводности с учетом случайного изменения коэффициента теплопроводности. Математическое моделирование проведено для взаимодействия широкого пучка импульсного лазерного излучения с поверхностью монокристаллической полупроводниковой мишени. Получена порядковая оценка погрешности для квадратичного функционала. Приведены результаты вычислительного эксперимента для параметров, характерных для теллурида кадмия.
\end{abstract}

Ключевые слова: уравнение теплопроводности, проекционный метод наименьших квадратов, функции Лагерра, математическое ожидание, автокорреляционная функция, модуль непрерывности.

\section{ON THE CALCULATION OF THE MOMENT FUNCTIONS OF A STOCHASTIC HEAT CONDUCTION PROCESS BY USING THE PROJECTION METHOD}

\author{
(c) 2021 E. V. SEREGINA, M. A. STEPOVICH, V. V. KALMANOVICH
}

\begin{abstract}
We present some results of using the projection method of least squares for finding the first- and second-order moment functions for a solution of the heat equation taking into account a random change in the coefficient of heat conductivity. Mathematical modeling was performed for the interaction of a wide beam of pulsed laser radiation with the surface of a single-crystal semiconductor target. An ordinal error estimate for a quadratic functional is obtained. The results of a computational experiment for the parameters characteristic of cadmium telluride are presented.
\end{abstract}

Keywords and phrases: heat equation, projection method of least squares, Laguerre functions, mathematical expectation, autocorrelation function, modulus of continuity.

AMS Subject Classification: 35G16, 33C10, 78A55

1. Введение. Анализ температурных полей, возникающих в результате облучения поверхности твердого тела ускоренными заряженными частицами или импульсами электромагнитного излучения может иметь важное практическое значение в различных отраслях науки и техники, в частности, в физическом материаловедении. При облучении образца в месте его воздействия на мишень в приповерхностной области мишени может выделяться значительная энергия, что ведет

Работа выполнена при поддержке Российского фонда фундаментальных исследований и правительства Калужской области (проекты № 19-03-00271 и № 18-41-400001). 
к повышению локальной температуры (см. [20,21,23]) и, как следствие, к изменению характеристик вещества, в некоторых случаях необратимому. Например, для материалов полупроводниковой микро- и наноэлектроники такое воздействие приводит к изменению ряда электрофизических параметров (см. [4]), например, ширины запрещенной зоны, что может привести к необходимости учета этого явления при эксплуатации приборных устройств (например, элементов солнечных батарей). Учет нагрева полупроводникового материала особенно существенен при использовании концентрированных источников воздействия, например, сфокусированных электронных пучков, широко используемых в электронно-зондовых технологиях (см. [3, 22, 23]).

При облучения поверхности твердого тела ускоренными заряженными частицами или электромагнитным излучением экспериментальное определение локальной температуры нагрева мишени сильно осложнено малым размером области генерации тепла: в начальный момент времени - порядка десятых или даже сотых долей микрометра (см. [8,23]), - а при импульсном воздействии еще и необходимостью учета зависимости процесса теплопередачи от времени, вследствие чего полученные результаты имеют невысокую точность и могут рассматриваться лишь как качественные. Поэтому особую ценность в практике воздействия заряженными частицами или импульсами электромагнитного излучения на твердые тела приобретают расчетные оценки величины нагрева, основанные на решении уравнения теплопроводности (см. [2,3,23]).

На практике локальные значения электрофизических параметров материалов в силу ряда причин могут иметь случайный разброс относительно своих усредненных по объему значений, и не исключено, что наличие разброса в значениях локальных параметров может оказать существенное влияние на процессы тепломассопереноса в таких материалах. Количественное описание влияния разброса в значениях локальных параметров материалов на их нагрев пучками заряженных частиц или импульсным электромагнитным излучением может быть проведено методами математического моделирования. Однако задача анализа моделей стохастических процессов, подобных процессам тепломассопереноса, с учетом случайного изменения электрофизических параметров исследуемого материала, является достаточно сложной проблемой, для решения которой существует сравнительно мало методов. Большинство таких методов являются либо слишком сложными для использования на практике, либо требуют принятия слишком грубых упрощающих допущений, например, о малости случайных возмущений параметров, при этом далеко не всегда удается найти точное аналитическое решение. В силу вышеизложенного разработка новых приближенно-аналитических методов моделирования стохастических процессов тепломассопереноса, ориентированных на использование ЭВМ, и создание эффективных вычислительных алгоритмов, является актуальной. В данной работе для исследования результатов стохастических процессов импульсного лазерного воздействия на мишени различной природы предлагается использовать проекционный метод, основанный на теории матричных операторов (см. [9]).

Ранее (см. [12]) с использованием проекционного метода было получено решение стационарного уравнения диффузии неравновесных неосновных носителей заряда, генерированных в полупроводниковой мишени киловольтными электронами. Приближенное решение было найдено в аналитическом виде с использованием сравнительно небольшого числа (15) членов функционального ряда.

Изучение возможностей проекционного метода для решения стационарных задач тепломассопереноса, обусловленного пучком ускоренных электронов или электромагнитным излучением, продолжено в [13-17]. В отличие от этих работ, объектом рассмотрения в настоящей работе является нестационарное уравнение теплопроводности, обусловленное воздействием на конденсированное вещество импульсного лазерного излучения, а модельные расчеты проведены для полупроводниковой мишени.

2. Постановка задачи. Рассмотрим дифференциальное уравнение теплопроводности, обусловленной воздействием широкого импульса лазерного излучения на полубесконечный полупроводник. В этом случае диаметр светового пучка будет много больше характерных размеров нагрева объемной части мишени, перпендикулярной плоской поверхности полупроводника, что 
позволяет пренебречь краевыми эффектами и записать соответствующее уравнение теплопереноса в следующем виде (см. [21]):

с граничными условиями

$$
\rho c \frac{\partial \Delta T}{\partial t}-\lambda \frac{\partial^{2} \Delta T}{\partial z^{2}}=\alpha(1-A) I_{0}(t) \exp (-\alpha z)
$$

$$
\Delta T(z, 0)=0, \quad \Delta T(+\infty, t)=0, \quad \Delta T(z,+\infty)=0, \quad 0 \leqslant z<\infty, \quad 0 \leqslant t<\infty .
$$

Здесь $\Delta T(z, t)=T(z, t)-T_{0}$, где $T$ - температура образца в точке $(z, t)$, обусловленная действием лазерного импульса, $T_{0}$ - начальная температура образца до воздействия лазерного импульса; $A$-коэффициент отражения и $\alpha$ - коэффициент поглощения лазерного излучения;

$$
I_{0}(t)=\frac{W}{6 \tau_{m}}\left(\frac{4 t}{\tau_{m}}\right)^{4} \exp \left(-\frac{4 t}{\tau_{m}}\right)
$$

- плотность потока энергии (см. [20]), где $W$ - плотность энергии лазерного импульса, $\tau_{m}$ - длительность переднего фронта импульса; $\lambda$ - коэффициент теплопроводности.

Поставим задачу определения математического ожидания и автокорреляционной функции изменения температуры $\Delta T(z, t)$, если коэффициент $\lambda$ испытывает флуктуации. Задача такого типа является важной для практики, т. к. многие теплотехнические объекты часто характеризуются нестабильностью параметров (см. [7]).

Существует несколько подходов к нахождению первых моментных функций решения дифференциальных уравнений со случайными коэффициентами. Математическое ожидание и вторая моментная функция решения задачи Коши для неоднородного уравнения теплопроводности со случайными коэффициентами могут быть найдены путем сведения к дифференциальным уравнениям с вариационными производными (см., например, [5]). В настоящей работе применяется проекционный метод для нахождения моментных функций температуры и решение ищется в виде рядов Фурье по модифицированным функциям Лагерра.

3. Проекционная аппроксимация исходной модели, основанная на применении метода наименьших квадратов. Согласно методу наименьших квадратов (MHK), вместо задачи (1)-(2) вводится в рассмотрение функционал

$$
J(\Delta T(z, t))=\left\|L^{(\Delta T)}(\Delta T(z, t))-f(z, t)\right\|_{L_{2}}^{2}+\|\Delta T(z, 0)\|_{L_{2}}^{2}+\|\Delta T(z,+\infty)\|_{L_{2}}^{2}+\|\Delta T(+\infty, t)\|_{L_{2}}^{2},
$$

где $L^{(\Delta T)}$ - дифференциальный оператор, стоящий в левой части уравнения (1),

$$
f(z, t)=\frac{\alpha}{\rho c}(1-A) I_{0}(t) \exp (-\alpha z)
$$

и $\Delta T(z, t)$ ищется из требования, чтобы оно доставляло минимум функционалу $J(\Delta T(z, t))$.

Поскольку граничное условие задано на бесконечности, введем двумерный базис из модифицированных функций Лагерра с параметрами, ускоряющими сходимость ряда:

$$
\varphi_{i, j}(z, t)=\varphi_{i}^{\alpha_{1}, \gamma_{1}}(z) \varphi_{j}^{\alpha_{2}, \gamma_{2}}(t)=\exp \left(-\gamma_{1} z / 2\right) L_{i}\left(\gamma_{1} z ; \alpha_{1}\right) \exp \left(-\gamma_{2} t / 2\right) L_{j}\left(\gamma_{2} t ; \alpha_{2}\right),
$$

которые определяются через многочлены Чебышева-Лагерра по переменной $z-L_{i}\left(\gamma_{1} z ; \alpha_{1}\right)$ и многочлены по переменной $t-L_{j}\left(\gamma_{2} t ; \alpha_{2}\right), i, j=0,1,2, \ldots$ Здесь параметры $\alpha_{1}>-1 / 2$ и $\alpha_{2}>-1 / 2$, а $\gamma_{1}>0$ и $\gamma_{2}>0$ используются для оптимизации вычислительной схемы (см. [18]).

Для простоты будем брать одинаковое число базисных функций. Тогда каждую функцию от переменных $z$ и $t$, входящую в исходную систему уравнений, аппроксимируем частичной суммой порядка $(m, m)$ ее двойного ряда Фурье-Лагерра и перейдем от системы уравнений (1)-(2) к приближенной системе уравнений. Приближенное решение $\Delta T_{m, m}(z, t)$ ищем из требования, чтобы оно доставляло минимум функционалу $J\left(\Delta T_{m, m}(z, t)\right)$.

Обозначим через $C_{m m}^{\Delta T}$ и $C_{m m}^{f}$ столбцы (матрицы, растянутые в столбцы) из коэффициентов разложения соответственно неизвестной функции $\Delta T(z, t)$ и известной $f(z, t)$ по двумерному базису $\varphi_{i}^{\alpha_{1}, \gamma_{1}}(z) \varphi_{j}^{\alpha_{2}, \gamma_{2}}(t)$.

Введем матрицы дифференцирования $D_{1}^{m}$ и $D_{2}^{m}$ в одномерных базисах $\varphi_{i}^{\alpha_{1}, \gamma_{1}}(z)$ и $\varphi_{j}^{\alpha_{2}, \gamma_{2}}(t)$ соответственно, и матрицы дифференцирования в пространствах вектор-функций $D^{t}=D_{2} \otimes E$, 
$D^{z}=E \otimes D_{1}$. Здесь $E$ - единичная матрица. Элементы матрицы дифференцирования $D$ в одномерном базисе находятся с помощью элементарных алгебраических операций (см. [9]):

$$
d_{i j}= \begin{cases}-\gamma / 2, & i=j, \\ -\gamma, & i<j, \\ 0, & i>j\end{cases}
$$

Обозначим через $\varphi_{m}^{\alpha_{1}, \gamma_{1}}(z)$ и $\varphi_{m}^{\alpha_{2}, \gamma_{2}}(t)$ столбцы из $m$ первых базисных функций по переменным $z$ и $t$ соответственно. В силу ортогональности базисных функций функционал $J\left(\Delta T_{m, m}(z, t)\right)$ можно представить в виде:

$$
J\left(C^{\Delta T}\right)=\left(A C^{\Delta T}-G\right)^{T}\left(A C^{\Delta T}-G\right) \rightarrow \inf ,
$$

где

$$
A=\left[\begin{array}{c}
D^{t}-\lambda /(\rho c)\left(D^{z}\right)^{2} \\
\left(\varphi_{m}^{\alpha_{2}, \gamma_{2}}(0)\right)^{T} \otimes E
\end{array}\right], \quad G=\left[\begin{array}{c}
C_{m m}^{f} \\
O_{m \times 1}
\end{array}\right]
$$

Тогда нормальное псевдорешение находим по формуле

$$
C^{\Delta T^{+}}=\left(A^{T} A\right)^{-1} A^{T} G
$$

Получив нормальное псевдорешение $C^{\Delta T^{+}}$, восстанавливаем приближенное решение исходной задачи

$$
\Delta T(z, t) \approx \Delta T_{m, m}(z, t)=\left(\varphi_{m}^{\alpha_{2}, \gamma_{2}}(t)\right)^{T} C^{\Delta T^{+}} \varphi_{m}^{\alpha_{1}, \gamma_{1}}(z), \quad z \in[0, \infty), \quad t \in[0, \infty) .
$$

4. Условие сходимости. Следуя [1], введем обозначения

$$
D=z \frac{d^{2}}{d z^{2}}+\left(\alpha_{1}-z+1\right) \frac{d}{d z}+t \frac{d^{2}}{d t^{2}}+\left(\alpha_{2}-t+1\right) \frac{d}{d t} .
$$

Здесь $L_{2}^{n}(D)(n=0,1, \ldots)$ - класс таких функций $f$, что функции

$$
\tilde{f}(z, t)=f(z, t) \exp \left(\gamma_{1} z / 2\right) \exp \left(\gamma_{2} t / 2\right) \in L_{2}
$$

и имеющие обобщенные частные производные в смысле Леви (см. [1]) $\partial^{k} / \partial z^{i} \partial t^{j} \tilde{f}(z, t), i+j=k$, $k=0,1, \ldots$, принадлежащие пространству $L_{2}$, для которых $D^{n} \tilde{f} \in L_{2}, n=0,1, \ldots$, где $D_{2}^{0} \tilde{f}=\tilde{f}$, $D^{n} \tilde{f}=D\left(D^{n-1} \tilde{f}\right), n=1,2, \ldots, L_{2}^{0}(D)=L_{2}$.

Если погрешности в исходных данных и погрешности вычислений отсутствуют, а учитываются лишь погрешности аппроксимаций, то имеет место следующая теорема.

Теорема 1. Пусть функиия $f(z, t) \in L_{2}^{n}(D)$ и имеет непрерывные производные до порядка $n>\alpha_{2} / 2+3 / 4$ по обоим пространственным направлениям. Тогда последовательность нормальных псевдорешений $\left\{C_{m m}^{\Delta T^{+}}\right\}$будет минимизирующей для функционала $J\left(C_{m m}^{\Delta T}\right)$ и справедлива оченка

$\sqrt{J\left(C_{m m}^{\Delta T^{+}}\right)}<C\left[1-\left(1-m^{-1 / 2}\right)^{m}\right]^{-k}(m)^{-n} \Omega\left(m^{-1 / 2}\right)+O\left(m^{-n+\alpha_{2} / 2+3 / 4}\right) \omega\left(m^{-1 / 2}\right), \quad m \rightarrow \infty$, где положительная постоянная $C$ не зависит от $m$, а $\Omega\left(m^{-1 / 2}\right)$ - мажсоранта обобщенных модулей непрерывности и $\omega\left(m^{-1 / 2}\right)$ - заданная мажсоранта модулей непрерывности.

Доказательство. Для точного решения имеем

$$
\frac{\partial \Delta T}{\partial t}-\frac{\lambda}{\rho c} \frac{\partial^{2} \Delta T}{\partial z^{2}}=f(z, t)
$$

Тогда

$$
\begin{gathered}
\sqrt{J\left(C_{m m}^{\Delta T^{+}}\right)} \leqslant \sqrt{J\left(C_{m m}^{\Delta T}\right)} \leqslant\left\|\frac{\partial \Delta T(z, t)}{\partial t}-\frac{\partial \Delta T_{m, m}(z, t)}{\partial t}\right\|_{L_{2}}+ \\
+\frac{\lambda}{\rho c}\left\|\left(\frac{\partial^{2} \Delta T(z, t)}{\partial z^{2}}-\frac{\partial^{2} \Delta T_{m, m}(z, t)}{\partial z^{2}}\right)\right\|_{L_{2}}+\left\|f(z, t)-f_{m, m}(z, t)\right\|_{L_{2}}+\left\|\Delta T(z, 0)-\Delta T_{m, m}(z, 0)\right\|_{L_{2}} .
\end{gathered}
$$


Обозначим слагаемые в правой части последнего неравенства через $S_{1}, \ldots, S_{4}$ и воспользуемся оценками из $[1,10,11]$, т.е.

$$
\begin{aligned}
& S_{1}=\left\|\frac{\partial \Delta T(z, t)}{\partial t}-\frac{\partial \Delta T_{m, m}(z, t)}{\partial t}\right\|_{L_{2}} \leqslant \\
& \leqslant\left[1-(1-h)^{m}\right]^{-k-1} m^{-n} \Omega_{k+1}\left(D^{n}\left(\Delta \tilde{T}_{t}^{\prime}(z, t)+\frac{\gamma_{2}}{2} \Delta \tilde{T}(z, t)\right) ; h\right)+ \\
& +\left[1-(1-h)^{m}\right]^{-k-2} m^{-n-1} \Omega_{k+2}\left(D^{n+1}\left(\frac{\gamma_{2}}{2} \Delta \tilde{T}(z, t)\right) ; h\right), \\
& S_{2}=\frac{\lambda}{\rho c}\left\|\left(\frac{\partial^{2} \Delta T(z, t)}{\partial z^{2}}-\frac{\partial^{2} \Delta T_{m, m}(z, t)}{\partial z^{2}}\right)\right\|_{L_{2}} \leqslant \\
& \leqslant \frac{\lambda}{\rho c}\left(\left[1-(1-h)^{m}\right]^{-k} m^{-n} \Omega_{k}\left(D^{n}\left(\Delta \tilde{T}_{z z}^{\prime \prime}(z, t)+\gamma_{1} \Delta \tilde{T}_{z}^{\prime}(z, t)+\frac{\gamma_{1}^{2}}{4} \Delta \tilde{T}(z, t)\right) ; h\right)+\right. \\
& +\left[1-(1-h)^{m}\right]^{-k-1} m^{-n} \Omega_{k+1}\left(D^{n}\left(\gamma_{1} \Delta \tilde{T}_{z}^{\prime}(z, t)+\frac{\gamma_{1}^{2}}{2} \Delta \tilde{T}(z, t)\right) ; h\right)+ \\
& \left.+\left[1-(1-h)^{m}\right]^{-k-2} m^{-n-1} \Omega_{k+2}\left(D^{n+1}\left(\frac{\gamma_{1}^{2}}{4} \Delta \tilde{T}(z, t)\right) ; h\right)\right), \\
& S_{3}=\left\|f(z, t)-f_{m, m}(z, t)\right\|_{L_{2}} \leqslant\left[1-(1-h)^{m}\right]^{-k-2} m^{-n-1} \Omega_{k+2}\left(D^{n+1}(\tilde{f}(z, t)) ; h\right), \\
& S_{4}=\left\|\Delta T(z, 0)-\Delta T_{m, m}(z, 0)\right\|_{L_{2}} \leqslant \\
& \leqslant\left[1-(1-h)^{m}\right]^{-k-2} m^{-n-1} \Omega_{k+2}\left(D^{n+1}(\Delta \tilde{T}(z, 0)) ; h\right)+O\left(m^{-n+\alpha_{2} / 2+3 / 4}\right) \omega\left(m^{-1 / 2}\right) .
\end{aligned}
$$

Последняя оценка имеет место, если $n>\alpha_{2} / 2+3 / 4$. Ясно, что если функция бесконечное число раз непрерывно дифференцируема, то полученная оценка справедлива при любом $n>\alpha_{2} / 2+3 / 4$.

Полагая $h=m^{-1 / 2}$ и собрав все оценки вместе, получаем оценку для функционала:

$$
\sqrt{J\left(C_{m m}^{\Delta T^{+}}\right)}<C\left[1-\left(1-m^{-1 / 2}\right)^{m}\right]^{-k} m^{-n} \Omega\left(m^{-1 / 2}\right)+O\left(m^{-n+\frac{\alpha_{2}}{2}+\frac{3}{4}}\right) \omega\left(m^{-1 / 2}\right) ;
$$

здесь $\Omega\left(m^{-1 / 2}\right)$ - мажоранта обобщенных модулей непрерывности, а $\omega\left(m^{-1 / 2}\right)$ - заданная мажоранта модулей непрерывности (см. [1]). Теорема доказана.

5. Использования проекционной аппроксимации для нахождения моментных функций. Пусть $\lambda$-случайная величина, распределенная по нормальному усеченному закону с заданным математическим ожиданием $m_{\lambda}$ и средним квадратическим отклонением $\sigma_{\lambda}$. Тогда выражение (4) можно записать в виде:

$$
W C^{\Delta T}=Y
$$

где $W=A^{T} A, Y=A^{T} G$. Матрицы $W$ и $Y$ имеют следующую структуру:

$$
W=\lambda^{2}(r) W_{1}+\lambda(r) W_{2}+W_{3}, \quad Y=\lambda(r) Y_{1}+Y_{2},
$$

где $W_{1}, W_{2}, W_{3}, Y_{2}$ и $Y_{2}$ - детерминированные матрицы, а $\lambda(r)=m_{\lambda}+r \sigma_{\lambda}, r$ - непрерывная случайная величина, распределенная по нормальному усеченному закону, и имеющая нулевое математическое ожидание и единичную дисперсию.

Из выражения (4) находится проекционная характеристика математического ожидания решения задачи (1), (2):

$$
C^{m_{\Delta T^{+}}}=M\left[C^{\Delta T^{+}}(\lambda)\right]=\int_{\lambda_{1}}^{\lambda_{2}} C^{\Delta T^{+}}(\lambda) f(\lambda) d \lambda=M\left[(W(\lambda))^{-1} Y(\lambda)\right] .
$$


Здесь функция $f(\lambda)$ представляет собой плотность нормального усеченного закона распределения случайной величины $\lambda$, ее возможные значения принадлежат промежутку $\lambda \in\left[\lambda_{1}, \lambda_{2}\right]$.

Для того чтобы найти математическое ожидание от обратной случайной матрицы, умноженной на некоторую случайную матрицу $Y$, целесообразно предварительно подготовить систему (5) к разложению в сходящийся матричный ряд (см. [19]). Затем перейдем от системы (5) к равносильной системе

$$
H W_{0}(r) W(r) C^{\Delta T}=H W_{0}(r) Y(r) .
$$

Здесь $W_{0}(r)=\exp \left(-r^{2} / \mu\right) \cdot(W(0))^{-1}, \mu>0$ - параметр оптимизации, $H=2 E /(\widehat{M}+\widehat{m})$, где

$$
\widehat{m}=\min _{j}\left(\min _{r}\left(\lambda_{j}\left(W_{0}(r) W(r)\right)\right)\right), \quad \widetilde{M}=\max _{j}\left(\max _{r}\left(\lambda_{j}\left(W_{0}(r) W(r)\right)\right)\right),
$$

a $\lambda_{j}\left(W_{0}(r) W(r)\right)$ - собственные значения матрицы $W_{0}(r) W(r)$, т.е. спектр

$$
\operatorname{Sp}\left(W_{0}(r) W(r)\right) \in[\widehat{m}, \widehat{M}]
$$

(см. $[6,16])$. Тогда почти все собственные значения матрицы $\widehat{W}(r)=E-H W_{0}(r) W(r)$ будут заключены в открытом интервале (см. $[6,16])$. Ряд

$$
(E-\widehat{W}(r))^{-1}=\sum_{\nu=0}^{\infty}(\widehat{W}(r))^{\nu}
$$

можно умножить на ограниченную функцию $f(\lambda)$, представляющую собой плотность распределения случайной величины $\lambda$ и проинтегрировать в пределах от $\lambda_{1}$ до $\lambda_{2}$ (см. [6]). Тогда проекционные характеристики математического ожидания и автокорреляционной функции решения уравнения (1), (2) могут быть найдены с помощью следующих разложений:

$$
\begin{gathered}
C^{m_{\Delta T^{+}}}=\sum_{\nu=0}^{\infty} M\left[(\widehat{W}(r))^{\nu} H W_{0}(r) Y(r)\right] \\
C^{R_{\Delta T^{+}}}=\sum_{\nu_{1}=0}^{\infty} \sum_{\nu_{2}=0}^{\infty} M\left[(\widehat{W})^{\nu_{1}} H W_{0} Y Y^{T} W_{0}^{T} H^{T}\left((\widehat{W})^{T}\right)^{\nu_{2}}\right]-C^{m_{\Delta T^{+}}}\left(C^{m_{\Delta T^{+}}}\right)^{T} .
\end{gathered}
$$

Математическое ожидание и автокорреляционная функция температуры восстанавливаются по формулам:

$$
\begin{gathered}
m_{\Delta T}(z, t) \approx_{\nu} m_{\Delta T}(z, t) \approx\left(\varphi_{m}^{\alpha_{2}, \gamma_{2}}(t)\right)^{T} C_{\nu}^{m_{\Delta T^{+}}} \varphi_{m}^{\alpha_{1}, \gamma_{1}}(z), \quad z \in[0, \infty), \quad t \in[0, \infty) \\
R_{\Delta T}\left(t_{1}, t_{2} ; z_{1}, z_{2}\right) \approx_{\nu} R_{\Delta T}\left(t_{1}, t_{2} ; z_{1}, z_{2}\right) \approx\left(\varphi_{m}^{\alpha_{2}, \gamma_{2}}\left(t_{1}\right) \otimes \varphi_{m}^{\alpha_{1}, \gamma_{1}}\left(z_{1}\right)\right)^{T} \cdot C_{\nu}^{R_{\Delta T^{+}}}\left(\varphi_{m}^{\alpha_{2}, \gamma_{2}}\left(t_{2}\right) \otimes \varphi_{m}^{\alpha_{1}, \gamma_{1}}\left(z_{2}\right)\right),
\end{gathered}
$$
где $C_{\nu}^{m} \Delta T^{+}$и $C_{\nu}^{R_{\Delta T^{+}}}-\nu$-е приближения проекционных характеристик.

6. Результаты расчетов. Вычисления выполнялись в системе MATLAB (The MathWorks, Inc.) версии 7.5.0.342 на персональном компьютере со следующими характеристиками: процесcop Intel Pentium E5400 (2x2,70 GHz, 2 MB Cache), объем оперативной памяти - 2 GB.

Некоторые результаты детерминированного и статистического анализов представлены на рисунке. Расчеты проведены для параметров, характерных для полубесконечного монокристаллического теллурида кадмия: $c=0,205$ Дж/(г·K), $\lambda=0,057 \mathrm{Bт} /($ см·K $)$, коэффициента поглощения $\alpha=2 \times 10^{-3} \mathrm{~cm}^{-1}-$ и следующих параметров лазерного излучения: плотности энергии лазерного импульса $W=10$ кДж/ $\mathrm{cm}^{2}$, длительности переднего фронта импульса $\tau_{m}=12,605$ нс, длины волны излучения 10 мкм.

Оценка относительной погрешности по норме пространства $L_{2}$ :

$$
\Delta_{1}\left(\delta T_{7,7}, \delta T_{6,6}\right)=\frac{\left\|\delta T_{7,7}(z, t)-\delta T_{6,6}(z, t)\right\|_{L_{2}}}{\left\|\delta T_{7,7}(z, t)\right\|_{L_{2}}} \times 100 \%<3 \% .
$$

Если $\lambda$-случайная величина, распределенная по нормальному усеченному закону с математическим ожиданием $m_{\lambda}=0,057 \mathrm{BT} /(\mathrm{cm} \cdot \mathrm{K})$ и средним квадратическим отклонением 


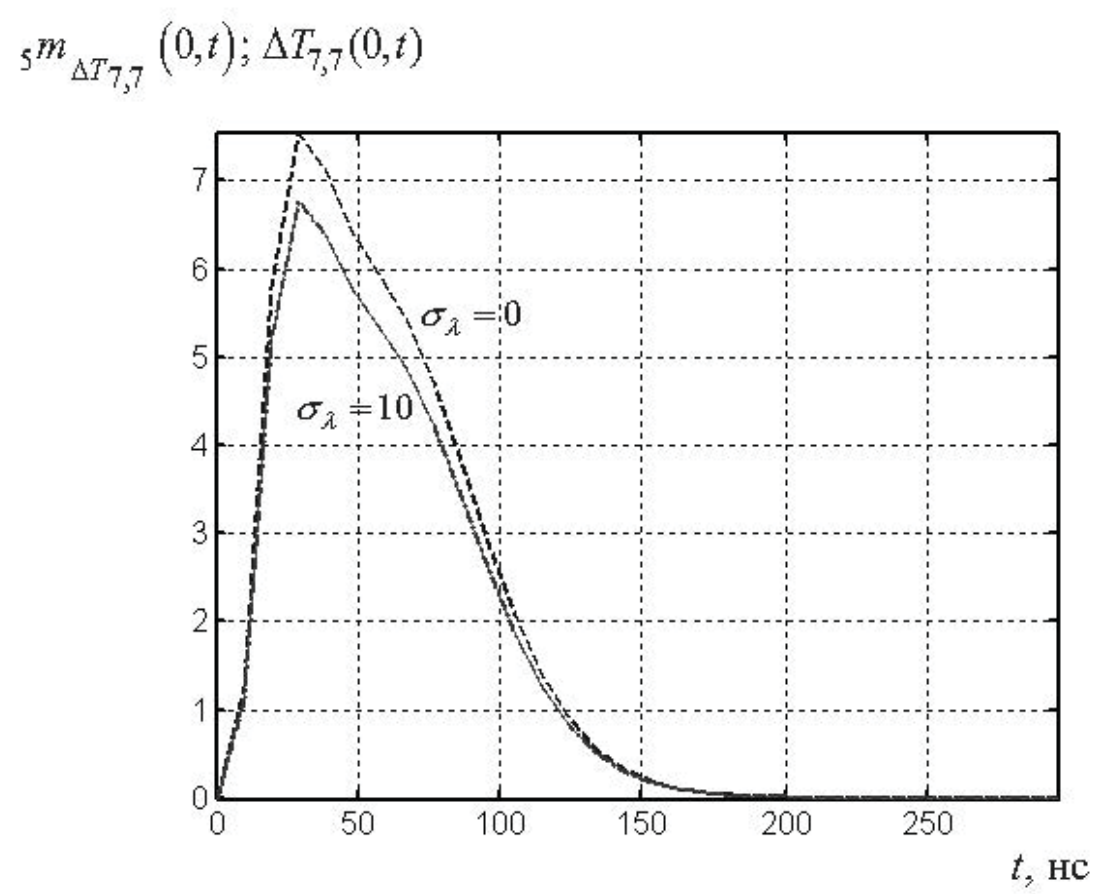

Рис. 1. Зависимость температуры на поверхности монокристаллического $\mathrm{Cd}$ Te (при $z=0$ ) от времени. График этой зависимости указан штриховой линией (верхний график), а его усредненное значение (математическое ожидание) указано непрерывной линией (нижний график).

$\sigma_{\lambda}=10 \mathrm{~B}$ / $\left(\right.$ см·K), возможные значения $\lambda$ принадлежат промежутку $\lambda \in\left[10^{-3}, 10^{3}\right]$ Вт/(см·K), тогда

$$
\begin{gathered}
\Delta_{2}\left({ }_{5} m_{\delta T_{7,7}}(z, t){ }_{4} m_{\delta T_{7,7}}(z, t)\right)<0,2 \%, \\
\Delta_{3}\left({ }_{5} R_{\delta T_{7,7}}\left(t_{1}, t_{2} ; z_{1}, z_{2}\right)_{4} R_{\delta T_{7,7}}\left(t_{1}, t_{2} ; z_{1}, z_{2}\right)\right)<3 \% .
\end{gathered}
$$

Из рис. 1 видно, что флуктуации параметра $\lambda$ замедляют темп разогрева. Такой же результат был получен в [7] для объекта конечной длины, а статистические характеристики температуры были найдены через функцию Грина.

7. Заключение. С использованием проекционного метода наименьших квадратов найдены моментные функции первого и второго порядка решения уравнения теплопроводности с учетом случайного изменения коэффициента теплопроводности. Математическое моделирование проведено для взаимодействия широкого пучка импульсного лазерного излучения с поверхностью монокристаллической полупроводниковой мишени. Получена порядковая оценка погрешности для квадратичного функционала. Затраты машинного времени на расчет моментных функций температуры с использованием проекционного метода наименьших квадратов составили приблизительно 3 с, что говорит о вычислительной эффективности предложенного метода.

\section{СПИСОК ЛИТЕРАТУРЫ}

1. Абилов В. А., Абилов М. В., Керимов М. К. Точные оценки скорости сходимости двойных рядов Фурье по классическим ортогональным многочленам// Ж. вычисл. мат. мат. физ. -2015 . -55 , № 7 . - C. 1109-1117. 
2. Амрастанов A. Н., Гинзгеймер C. A., Степович M. A., Филиппов М. Н. Об одной возможности математического моделирования теплового воздействия остро сфокусированного электронного пучка на однородный полупроводник// Изв. РАН. Сер. физ. - 2016. - 80, № 10. - С. 1448-1452.

3. Амрастанов A. Н., Серегина E. B., Степович M. А. Об одной особенности моделирования нагрева полупроводниковой мишени электронным зондом// Изв. РАН. Сер. физ. - 2018. - 82, № 9. — С. $1304-$ 1309.

4. Бонч-Бруевич В. Л., Калашников С. Г. Физика полупроводников. - М.: Наука, 1990.

5. Задорожний В. Г., Хребтова С. С. Первые моментные функции решения уравнения теплопроводности со случайными коэффициентами// Ж. вычисл. мат. мат. физ. - 2009. - 49, № 11. - С. $1937-1952$.

6. Корн Г., Корн Т. Справочник по математике для научных работников и инженеров. - М.: Наука, 1974.

7. Крушель Е. Г. Вероятностный анализ распределенных систем// в кн.: Нелинейные и оптимальные системы. - М.: Наука, 1971. - С. 36-43.

8. Кузин А. Ю., Степович М. А., Митюхляев В. Б., Тодуа П. А., Филиппов М. Н. Тепловые эффекты при низковольтном электронно-зондовом рентгеноспектральном микроанализе с нанометровой локальностью// Измерит. техн. - 2016. - 10. - С. 27-29.

9. Лапин C. B., Еәупов Н. Д. Теория матричных операторов и ее приложение к задачам автоматического управления. - М.: МГТУ им. Н. Э. Баумана, 1997.

10. Лащенов B. K. Приближение дифференцируемых функций частными суммами ряда ФурьеЛагерра// Изв. РАН. Мат. - 1981. - 1, № 224. - С. 44-57.

11. Макаренков A. М., Серегина E. B., Степович M. А. Проекционный метод Галеркина решения стационарного дифференциального уравнения диффузии в полубесконечной области// Ж. вычисл. мат. мат. физ. - 2017. - 57, № 5. - С. 801-813.

12. Петров В. И., Самохвалов А. А., Степович М. А., Чайковский М. М. Матричный метод решения задачи коллективного движения неосновных носителей заряда, генерированных в полупроводниковом материале электронным пучком// Изв. РАН. Сер. физ. - 2002. - 66, № 9. - С. 1310-1316.

13. Серегина Е. В., Макаренков А. М., Степович М. А. Использование проекционного метода для определения статистических характеристик решения дифференциального уравнения диффузии неосновных носителей заряда, генерированных в полупроводниковом материале широким электронным пучком// Поверхн. Рентген. синхротрон. нейтрон. исслед. - 2009. - 6. - С. 80-95.

14. Серегина E. B., Макаренков A. М., Степович M. А. О возможности реализации стохастической модели распределения неравновесных неосновных носителей заряда в полупроводниковом материале// Поверхн. Рентген. синхротрон. нейтрон. исслед. - 2009. - 10. - С. 75-86.

15. Серегина E. B., Макаренков A. M., Степович M. А. Статистический анализ модели коллективного движения неосновных носителей заряда, генерированных электронным пучком в полупроводниковом материале, с использованием проекционного метода// Поверхн. Рентген. синхротрон. нейтрон. исслед. - 2011. - 8. - C. 41-49.

16. Серегина E. B., Макаренков A. M., Степович M. А. Статистический анализ модели коллективного движения неосновных носителей заряда с использованием проекционного метода// Поверхн. Рентген. синхротрон. нейтрон. исслед. - 2012. - 4. - С. 47-55.

17. Серегина E. B., Cтепович M. A., Макаренков A. М. Об одной возможности статистического анализа распределения неосновных носителей заряда, генерированных электромагнитным излучением в полупроводниковом материале// Прикл. физ. - 2012. - 3. - С. 24-31.

18. Суетин П. К. Классические ортогональные многочлены. - М.: Физматлит, 2007.

19. Фадеев Д. К., Фадеева В. Н. Вычислительные методы линейной алгебры. - М.: Физматгиз, 1960.

20. Ханефт A. В., Долгачев В. А., Дугинов E. В., Иванов Г. А. Критерии зажигания энергетических материалов коротким лазерным и электронным импульсами// Вестн. Кемеровск. гос. ун-та - 2013. - 3, № 55. - C. 31-39.

21. Seregina E. V., Stepovich M. A., Kalmanovich V. V. Modeling of heating in the epitaxial structure $\mathrm{Cd}_{x} \mathrm{Hg}_{1-x} \mathrm{Te} / \mathrm{CdTe}$ with the projection least squares method// J. Phys. Conf. Ser. — 2019. — 1163. — P. 012013-1-012013-6.

22. Stepovich M. A., Amrastanov A. N., Seregina E. V., Filippov M. N. On one peculiarity of the model describing the interaction of the electron beam with the semiconductor surface// J. Phys. Conf. Ser. 2018. - 955. - P. 012040-1-012040-6. 
23. Stepovich M. A., Amrastanov A. N., Seregina E. V., Filippov M. N. Assessment of the heating of conductive targets with an electron beam. Results of computational experiment// J. Phys. Conf. Ser. — 2019. — 1203. - P. 012042-1-012042-8.

Серегина Елена Владимировна

Московский государственный технический университет им. Н. Э. Баумана

(национальный исследовательский университет), Калужский филиал

E-mail: evfs@yandex.ru

Степович Михаил Адольфович

Калужский государственный университет им. К. Э. Циолковского

E-mail: m.stepovich@rambler.ru

Калманович Вероника Валерьевна

Калужский государственный университет им. К. Э. Циолковского

E-mail: v572264@yandex.ru 